\title{
Monotone-Iterative Method for Solving Antiperiodic Nonlinear Boundary Value Problems for Generalized Delay Difference Equations with Maxima
}

\author{
Angel Golev, ${ }^{1}$ Snezhana Hristova, ${ }^{1}$ and Svetoslav Nenov ${ }^{2}$ \\ ${ }^{1}$ Faculty of Mathematics and Informatics, Plovdiv University, Tzar Asen 24, 4000 Plovdiv, Bulgaria \\ ${ }^{2}$ Department of Mathematics, University of Chemical Technology and Metallurgy, Kl. Ohridski 8, 1756 Sofia, Bulgaria \\ Correspondence should be addressed to Snezhana Hristova; snehri@gmail.com
}

Received 26 February 2013; Revised 30 June 2013; Accepted 17 July 2013

Academic Editor: Ferhan M. Atici

Copyright (C) 2013 Angel Golev et al. This is an open access article distributed under the Creative Commons Attribution License, which permits unrestricted use, distribution, and reproduction in any medium, provided the original work is properly cited.

A nonlinear generalized difference equation with both delays and the maximum value of the unknown function over a discrete past time interval are studied. A nonlinear boundary value problem of antiperiodic type for the given difference equation is set up. One of the main characteristics of the considered difference equation is the presence of the unknown function in both sides of the equation. It leads to impossibility for using the step method for explicit solving of the nonlinear difference equation. In this paper, an approximate method, namely, the monotone iterative technique, is applied to solve the problem. An important feature of the given algorithm is that each successive approximation of the unknown solution is equal to the unique solution of an appropriately constructed initial value problem for a linear difference equation with "maxima," and an algorithm for its explicit solving is given. Also, each approximation is a lower/upper solution of the given nonlinear boundary value problem. The suggested scheme for approximate solving is computer realized, and it is applied to a particular example, which is a generalization of a model in population dynamics.

\section{Introduction}

In the last few decades, great attention has been paid to automatic control systems and their applications to computational mathematics and modeling. Many problems in the control theory correspond to the maximal deviation of the regulated quantity. In the case when these problems are modeled discretely the corresponding equations are called difference equations with maxima. The presence of the maximum function in equations mostly requires new techniques for investigation of the qualitative behavior of their solutions. Note also differential equations with maxima are not well studied and still not widely applied as models (see, e.g., [1-5], the monograph [6], and cited therein references). The character of the maximum function leads to a variety of different types of difference equations. The properties of solutions of some special types of difference equations with maxima have been studied in [7-9]. However, such nonlinear difference equations can hardly be solved in explicit forms, and hence we need to develop some approximate methods for their solutions.

Note in several papers various types of boundary value problems for difference equations have been studied, and monotone iterative method has been applied. For example, in [10-13] first order difference equations have been studied in $[14,15]$ some criteria for existence and uniqueness results for $n$th order antiperiodic difference equations have been developed; in [16] a generalized delay difference equation is studied by lower and upper solutions, but the problem consists only of a boundary condition, which does not get uniqueness of the solution; the global boundary value problem for difference equations without any kind of delay is well studied by Cabada in [17]; in [18] a nonlinear boundary value problem for a delay difference equation with one delay is studied by monotone iterative method; in [19] an approximate method with a rapid convergence is applied to an initial value problem for difference equations with maxima, in [20] the monotoneiterative technique for nonlinear boundary value problem for 
difference equations with various delays as well as maxima is applied. Also, in [21], monotone iterative technique is applied to a periodic boundary value problem for difference equations with maxima but the successive approximation are solutions of periodic boundary value problems, which is practically difficult to be obtained.

However, in connection with the applications of difference equations as models of real world problems it is necessary to study difference equations with both delays and maximum over a discrete past time interval.

In this paper the authors consider a class of nonlinear difference equations with both delays and maximum of the unknown function over a discrete past time interval. A nonlinear boundary value problem of antiperiodic type is studied. An algorithm for constructing approximate solutions of this type of boundary value problem is developed. Any successive approximation is a solution of an initial value problem for a linear difference equation, and a practical algorithm for its obtaining is given and computer realized.

\section{Statement of the Problem}

Let $\mathbb{R}_{+}=[0, \infty), \mathbb{Z}$ be the set of all integers. For any $c, b \in \mathbb{Z}$ : $c<b$ we denote $\mathbb{Z}[c, b]=\{z \in \mathbb{Z}: c \leq z \leq b\}$.

Let $a, T \in \mathbb{Z}: T>a+1, r, p \in \mathbb{Z}[0, T-a]$, and $h \in \mathbb{Z}:$ $h>0$ be fixed.

Consider the following nonlinear mixed boundary value problem for the generalized nonlinear delay-difference equation with maxima (BVP)

$$
\begin{aligned}
& \Delta u(k-1) \\
& =f\left(k, u(k), u\left(\tau_{1}(k)\right), u\left(\tau_{2}(k)\right), \ldots,\right. \\
& \left.u\left(\tau_{r}(k)\right), \max _{s \in \mathbb{Z}[k-h, k]} u(s)\right), \quad \text { for } k \in \mathbb{Z}[a+1, T] \\
& g\left(u(a), u\left(T-\lambda_{1}\right), u\left(T-\lambda_{2}\right), \ldots, u\left(T-\lambda_{p}\right), u(T)\right)=0 \\
& u(k)=u(a) \quad \text { for } k \in \mathbb{Z}[a-h+1, a-1]
\end{aligned}
$$

where $u \in \mathbb{R}, \Delta u(k-1)=u(k)-u(k-1), f: \mathbb{Z}[a+1, T] \times$ $\mathbb{R}^{r+2} \rightarrow \mathbb{R}, g: \mathbb{R}^{p+2} \rightarrow \mathbb{R}, \tau_{m}(k): \mathbb{Z}[a+1, T] \rightarrow \mathbb{Z}[a+$ $1-h, T]: k-h \leq \tau_{m}(k) \leq k$ for $m=1,2, \ldots, r$.

Any solution of BVP (1)-(2) is a finite sequence of $T-a+h$ real numbers, and we will consider it as a real valued function with a discrete domain.

In this paper we will study boundary condition (2) in the case when it is from antiperiodic type. The studied problem includes as partial cases the initial value problem $(g(x) \equiv$ $x+$ const for $x \in \mathbb{R}$ ) and the antiperiodic boundary value problem $(g(x, u) \equiv x+u$ for $x, u \in \mathbb{R})$.

The presence of the delays $\tau_{m}$ generalizes the type of the considered difference equation since the function $f$ could depend on different delays at any point $k$. As a partial case $r=h$ and $\tau_{j}(k)=k-j, j=1, \ldots, r$ the right side of (1) is reduced to $f(k, u(k-h), u(k-h+1), \ldots, u(k-$ $\left.1), u(k), \max _{s \in \mathbb{Z}[k-h, k]} u(s)\right)$. For some other particular cases see Example 7 of the paper.

Note the explicit solving of BVP (1)-(2) is often impossible because of the nonlinearity and the presence of the unknown function $u(k), k \in \mathbb{Z}[a+1, T]$, in both sides of (1). It requires an application of approximate methods, one of which is an object of investigation in the paper.

\section{Preliminary Notes, Basic Notations, and Definitions}

Note, $\sum_{i=n}^{m} a_{i}=0$ and $\prod_{i=n}^{m} a_{i}=1$ where $m<n<\infty$ and $a_{j} \in \mathbb{R}, j \in Z[m, n]$.

For any function $u: \mathbb{Z}[a-h+1, T] \rightarrow \mathbb{R}$ we introduce the following notations:

$$
\begin{array}{r}
\chi(u(T))=\left(u\left(T-\lambda_{1}\right), u\left(T-\lambda_{2}\right), \ldots, u\left(T-\lambda_{p}\right), u(T)\right), \\
U(u(\tau(k)))=\left(u\left(\tau_{1}(k)\right), u\left(\tau_{2}(k)\right), \ldots, u\left(\tau_{r}(k)\right)\right), \\
k \in \mathbb{Z}[a+1, T], \\
\Xi(u(\tau(k)))=\left(u(k), U(u(\tau(k))), \max _{s \in \mathbb{Z}[k-h, k]} u(s)\right), \\
k \in \mathbb{Z}[a+1, T] .
\end{array}
$$

Using the previous notations the right side part of (1) could be written in a simpler way $f(k, \Xi(u(\tau(k))))$ and the nonlinear function in boundary condition (2) could be written as $g(u(a), \chi(u(T)))$.

Let $\alpha, \beta: \mathbb{Z}[a+1-h, T] \rightarrow \mathbb{R}$ be given functions such that $\alpha(k) \leq \beta(k)$ for $z \in \mathbb{Z}[a+1-h, T]$. Introduce the following sets:

$$
\begin{gathered}
S(\alpha, \beta) \\
=\{u: \mathbb{Z}[a-h+1, T] \longrightarrow \mathbb{R}: \alpha(k) \leq u(k) \leq \beta(k), \\
k \in \mathbb{Z}[a-h+1, T]\}, \\
W(\alpha, \beta)=\left\{x \in \mathbb{R}^{p+1}: \chi(\alpha(T)) \leq x \leq \chi(\beta(T))\right\}, \\
\Omega_{k}(\alpha, \beta)=\left\{V \in \mathbb{R}^{r+2}: \Xi(\alpha(\tau(k))) \leq V \leq \Xi(\beta(\tau(k)))\right\}, \\
k \in \mathbb{Z}[a+1, T] .
\end{gathered}
$$

Definition 1. The function $g(x, y) \in C([\alpha(a), \beta(a)] \times$ $W(\alpha, \beta), \mathbb{R})$ is said to be from the class $\Lambda(\alpha, \beta)$ if for $x \in$ $[\alpha(a), \beta(a)]$ and for $y_{1}, y_{2} \in W(\alpha, \beta): y_{1} \leq y_{2}$ the inequality $g\left(x, y_{1}\right) \leq g\left(x, y_{2}\right)$ holds and there exists a constant $\gamma>0$ such that for $u_{1}, u_{2} \in[\alpha(a), \beta(a)]: u_{2} \leq u_{1}$, the inequality $g\left(u_{1}, v\right)-g\left(u_{2}, v\right) \leq \gamma\left(u_{1}-u_{2}\right)$ holds for any $v \in W(\alpha, \beta)$.

Remark 2. Note if the function $g(x, y)$ is from the class $\Lambda(\alpha$, $\beta)$ then BVP (1)-(2) is from antiperiodic type. 
In connection with the construction of successive approximations we will introduce a couple of quasi-solutions of BVP (1)-(2).

Definition 3. One will say that the functions $\alpha, \beta \in \mathbb{Z}[a-h+$ $1, T]$ form a couple of quasi-solutions of BVP (1)-(2), if they satisfy (1) and

$$
\begin{aligned}
& g\left(\alpha(a), \beta\left(T-\lambda_{1}\right), \beta\left(T-\lambda_{2}\right), \ldots, \beta\left(T-\lambda_{p}\right), \beta(T)\right) \\
& \quad=0, \\
& g\left(\beta(a), \alpha\left(T-\lambda_{1}\right), \alpha\left(T-\lambda_{2}\right), \ldots, \alpha\left(T-\lambda_{p}\right), \alpha(T)\right) \\
& \quad=0, \\
& \alpha(k)=\alpha(a), \quad \beta(k)=\beta(a), \\
& \quad \text { for } k \in \mathbb{Z}[a-h+1, a-1] .
\end{aligned}
$$

Definition 4. One will say that the functions $\alpha, \beta \in \mathbb{Z}[a-h+$ $1, T]$ form a couple of quasi lower and upper solutions of BVP (1)-(2), if

$$
\begin{aligned}
& \Delta \alpha(k-1) \\
& \leq f\left(k, \alpha(k), \alpha\left(\tau_{1}(k)\right), \alpha\left(\tau_{2}(k)\right), \ldots,\right. \\
& \left.\quad \alpha\left(\tau_{r}(k)\right), \max _{s \in \mathbb{Z}[k-h, k]} \alpha(s)\right) \\
& \Delta \beta(k-1) \\
& \geq f\left(k, \beta(k), \beta\left(\tau_{1}(k)\right), \beta\left(\tau_{2}(k)\right), \ldots,\right. \\
& \left.\quad \beta\left(\tau_{r}(k)\right), \min _{s \in \mathbb{Z}[k-h, k]} \beta(s)\right) \text { for } k \in \mathbb{Z}[a+1, T], \\
& \left.\quad \leq 0, \quad \beta\left(T-\lambda_{1}\right), \beta\left(T-\lambda_{2}\right), \ldots, \beta\left(T-\lambda_{p}\right), \beta(T)\right) \\
& g\left(\beta(a), \alpha\left(T-\lambda_{1}\right), \alpha\left(T-\lambda_{2}\right), \ldots, \alpha\left(T-\lambda_{p}\right), \alpha(T)\right) \\
& \quad \geq 0, \\
& \alpha(k)=\alpha(a), \quad \beta(k)=\beta(a), \\
& \quad \text { for } k \in \mathbb{Z}[a-h+1, a-1] .
\end{aligned}
$$

\section{Linear Delay Difference Inequalities with Maxima}

One will consider the following linear difference inequality with maxima

$$
\begin{aligned}
\Delta u(k-1) \leq & -Q(k) u(k)-\sum_{j=1}^{r} C_{j}(k) u\left(\tau_{j}(k)\right) \\
& -q(k) \min _{s \in \mathbb{Z}[k-h, k]} u(s), \quad k \in \mathbb{Z}[a+1, T], \\
u(k)= & u(a) \leq 0, \quad k \in \mathbb{Z}[a-h+1, a-1] .
\end{aligned}
$$

Lemma 5 (comparison result). Let the function $u: \mathbb{Z}[a-h+$ $1, T] \rightarrow \mathbb{R}$ satisfy inequalities (7) and

$$
\sum_{l=a+1}^{T}\left(Q(l)+\sum_{j=1}^{r} C_{j}(l)+q(l)\right) \leq 1,
$$

where $Q, q, C_{j}: \mathbb{Z}[a+1, T] \rightarrow \mathbb{R}_{+}$and $\tau_{j}(k): \mathbb{Z}[a+1, T] \rightarrow$ $\mathbb{Z}[a+1-h, T]: k-h \leq \tau_{j}(k) \leq k$ for $j \in \mathbb{Z}[1, r], k \in$ $\mathbb{Z}[a+1, T]$.

Then $u(t) \leq 0$ for $t \in \mathbb{Z}[a-h+1, T]$.

Proof. Assume that the claim of Lemma 5 is not true. Consider the following two cases.

Case 1. Let $u(a)<0$. Therefore, there exists $\eta \in \mathbb{Z}[a+1, T]$ such that $u(k) \leq 0$ for $k \in \mathbb{Z}[a-h+1, \eta-1]$ and $u(\eta)>0$.

Denote $\min _{s \in \mathbb{Z}[a-h+1, \eta]} u(s)=-\lambda<0$. Let $\varsigma \in \mathbb{Z}[a, \eta]$, $\varsigma<\eta$, be such that $u(\varsigma)=-\lambda$. Then $u\left(\tau_{j}(l)\right) \geq-\lambda, j \in \mathbb{Z}[1, r]$, and $\min _{s \in \mathbb{Z}[l-h, l]} u(s) \geq-\lambda$ for all $l \in \mathbb{Z}[\varsigma+1, \eta]$.

Therefore,

$$
\begin{aligned}
\lambda< & u(\eta)-u(\varsigma) \\
= & (u(\eta)-u(\eta-1))+(u(\eta-1)-u(\eta-2)) \\
& +\cdots+(u(\varsigma+1)-u(\varsigma)) \\
= & \Delta u(\eta-1)+\Delta u(\eta-2)+\cdots+\Delta u(\varsigma) \\
\leq & \sum_{l=\varsigma+1}^{\eta}\left(-Q(l) u(l)-\sum_{j=1}^{r} C_{j}(l) u\left(\tau_{j}(l)\right)\right. \\
& \left.\quad-q(l) \min _{s \in \mathbb{Z}[l-h, l]} u(s)\right) \\
\leq & \lambda \sum_{l=c+1}^{\eta}\left(Q(l)+\sum_{j=1}^{r} C_{j}(l)+q(l)\right) \\
\leq & \lambda \sum_{l=a+1}^{T}\left(Q(l)+\sum_{j=1}^{r} C_{j}(l)+q(l)\right) .
\end{aligned}
$$

Inequality (9) contradicts (8).

Case 2. Let $u(a)=0$. Then there exists $\eta \in \mathbb{Z}[a, T-1]$ such that $u(k)=0$ for $k \in \mathbb{Z}[a+1-h, \eta]$ and $u(\eta+1) \neq 0$.

Let $u(\eta+1)<0$. As in the proof of Case 1 for $a=\eta+1$ we get a contradiction.

Let $u(\eta+1)>0$. Introduce the following sets:

$$
\begin{gathered}
\Omega_{\eta}=\left\{j \in \mathbb{Z}[1, r]: \tau_{j}(\eta+1)=\eta+1\right\}, \\
\bar{\Omega}_{\eta}=\left\{j: j \in \mathbb{Z}[1, r], j \notin \Omega_{\eta}\right\} .
\end{gathered}
$$


Note that the set $\Omega_{\eta}$ could be empty. Therefore, $u\left(\tau_{j}(\eta+\right.$ 1)) $=u(\eta+1)>0$ for $j \in \Omega_{\eta}$ and $u\left(\tau_{j}(\eta+1)\right)=0$ for $j \in \bar{\Omega}_{\eta}$. Then from inequality (7) we get

$$
\begin{gathered}
\left(1+Q(\eta+1)+\sum_{j \in \Omega_{\eta}} C_{j}(\eta+1)\right) u(\eta+1) \\
\leq-q(\eta+1) \min \{0, u(\eta+1)\}=0 .
\end{gathered}
$$

The obtained contradiction proves $u(\eta+1)<0$.

\section{Linear Delay Difference Equations with Maxima}

In connection with the computer realization of the suggested method, we will give an algorithm for exact solving of the initial value problem for the scalar linear delay-difference equation with maxima (IVP)

$$
\begin{aligned}
& \Delta u(k-1) \\
& =-Q(k) u(k)-q(k) \max _{s \in \mathbb{Z}[k-h, k]} u(s) \\
& \quad-\sum_{j=1}^{r} C_{j}(k) u\left(\tau_{j}(k)\right)+P(k), \quad k \in \mathbb{Z}[a+1, T], \\
& \quad u(k)=u(a), \quad k \in \mathbb{Z}[a-h+1, a-1],
\end{aligned}
$$

where $P: \mathbb{Z}[a+1, T] \rightarrow \mathbb{R}, Q, q, C_{j}: \mathbb{Z}[a+1, T] \rightarrow \mathbb{R}_{+}$, and $\tau_{j}(k): \mathbb{Z}[a+1, T] \rightarrow \mathbb{Z}[a+1-h, T]: k-h \leq \tau_{j}(k) \leq k$ for $j \in \mathbb{Z}[1, r], k \in \mathbb{Z}[a+1, T]$.

Note that the unknown function $u(k)$ at any moment $k \in$ $\mathbb{Z}[a+1, T]$ is included in the maximum function of the right side part of (12) and it is not obvious to solve (12). We will give the algorithm for obtaining the solution.

We will consider two cases with respect to the type of delays $\tau_{j}$.

Case 1. Let the inequality $\tau_{j}(k)<k$ hold for all $j \in \mathbb{Z}[1, r]$, $k \in \mathbb{Z}[a+1, T]$.

Assume that the values $u(k)$ of the unknown solution are obtained for all $k \in \mathbb{Z}[a-h+1, m]$, where $m<T$. Now let $k=m+1$.

Case 1.1. Let the following inequality be satisfied:

$$
\begin{gathered}
\frac{P(k)-\sum_{j=1}^{r} C_{j}(k) u\left(\tau_{j}(k)\right)+u(k-1)}{1+Q(k)+q(k)} \\
\geq \max \{u(k-l): l=1,2, \ldots, h\} .
\end{gathered}
$$

Therefore, $\max _{s \in \mathbb{Z}[k-h, k]} u(s)=u(k)$. Then the unique solution of IVP (12)-(13) is given by

$$
u(k)=\frac{P(k)-\sum_{j=1}^{r} C_{j}(k) u\left(\tau_{j}(k)\right)+u(k-1)}{1+Q(k)+q(k)} .
$$

Case 1.2. Let inequality (14) be not satisfied for all $l \in$ $\mathbb{Z}[1, h]$. Therefore, there exists $m \in \mathbb{Z}[1, h]$ such that $\max _{s \in \mathbb{Z}[k-h, k]} u(s)=u(k-m)$.

Then the unique solution of IVP (12)-(13) is

$u(k)$

$$
=\frac{P(k)-\sum_{j=1}^{r} C_{j}(k) u\left(\tau_{j}(k)\right)+u(k-1)-q(k) u(k-m)}{1+Q(k)} .
$$

Case 2. Let there exists at least one $j \in \mathbb{Z}[1, r]$ and $k \in \mathbb{Z}[a+$ $1, T]$ such that $\tau_{j}(k)=k$.

Assume that the values $u(k)$ of the unknown solution are obtained for all $k \in \mathbb{Z}[a-h+1, m]$, where $m<T$.

Now let $k=m+1$. Introduce the following sets:

$$
\begin{aligned}
& \Omega_{k}=\left\{j \in \mathbb{Z}[1, r]: \tau_{j}(k)=k\right\}, \\
& \bar{\Omega}_{k}=\left\{j: j \in \mathbb{Z}[1, r], j \notin \Omega_{k}\right\} .
\end{aligned}
$$

Note that the set $\Omega_{k}$ could be empty. Therefore, $u\left(\tau_{j}(k)\right)=$ $u(k)$ for $j \in \Omega_{k}$ and $u\left(\tau_{j}(k)\right)<u(k)$ for $j \in \bar{\Omega}_{k}$. Equation (12) is reduced to

$$
\begin{aligned}
\left(1+Q(k)+\sum_{j \in \Omega_{k}} C_{j}(k)\right) u(k) \\
=u(k-1)-\sum_{j \in \bar{\Omega}_{k}} C_{j}(k) u\left(\tau_{j}(k)\right)-q(k) \max _{s \in \mathbb{Z}[k-h, k]} u(s) \\
\quad+P(k) .
\end{aligned}
$$

Case 2.1. Let the following inequality be satisfied

$$
\begin{gathered}
\frac{P(k)+u(k-1)-\sum_{j \in \bar{\Omega}_{k}} C_{j}(k) u\left(\tau_{j}(k)\right)}{1+Q(k)+\sum_{j \in \Omega_{k}} C_{j}(k)+q(k)} \\
\geq u(k-s), \quad s \in \mathbb{Z}[1, h] .
\end{gathered}
$$

Therefore, $\max _{s \in \mathbb{Z}[k-h, k]} u(s)=u(k)$ and from (18) we obtain the solution

$$
u(k)=\frac{P(k)-\sum_{j \in \bar{\Omega}_{k}} C_{j}(k) u\left(\tau_{j}(k)\right)+u(k-1)}{1+Q(k)+\sum_{j \in \Omega_{k}} C_{j}(k)+q(k)} .
$$

Case 2.2. Let inequality (19) be not satisfied for all $s \in \mathbb{Z}[1, h]$.

Therefore, there exists $m \in \mathbb{Z}[1, h]$ such that $\max _{s \in \mathbb{Z}[k-h, k]} u(s)=u(k-m)$.

Then the unique solution of problem (12)-(13) is given by

$u(k)$

$$
=\frac{P(k)-\sum_{j \in \bar{\Omega}_{k}} C_{j}(k) u\left(\tau_{j}(k)\right)+u(k-1)-q(k) u(k-m)}{1+Q(k)+\sum_{j \in \Omega_{k}} C_{j}(k)} .
$$




\section{Monotone-Iterative Method}

We will give an algorithm for approximatly obtaining the solution of the mixed boundary value problem for the nonlinear delay-difference equation with maxima (1)-(2) as a limit of two monotone sequences.

Theorem 6. Let the following conditions be fulfilled:

(1) The functions $\alpha_{0}, \beta_{0}: \mathbb{Z}[a-h+1, T] \rightarrow \mathbb{R}$ form $a$ couple of quasi lower and upper solutions of (1)-(2) such that $\alpha_{0}(k) \leq \beta_{0}(k)$ for $k \in \mathbb{Z}[a-h+1, T]$.

(2) The function $f: \mathbb{Z}[a+1, T] \times \mathbb{R}^{r+2} \rightarrow \mathbb{R}$ is continuous in $\Omega_{k}\left(\alpha_{0}, \beta_{0}\right)$ for any $k \in \mathbb{Z}[a+1, T]$ and for $x, y \in \Omega_{k}\left(\alpha_{0}, \beta_{0}\right), x=\left(x_{1}, x_{2}, \ldots, x_{r+2}\right), y=$ $\left(y_{1}, y_{2}, \ldots, y_{r+2}\right)$ with $x_{j} \leq y_{j}, j \in \mathbb{Z}[1, r+2]$ the inequality

$$
\begin{aligned}
& f\left(k, x_{1}, x_{2}, \ldots, x_{r+2}\right)-f\left(k, y_{1}, y_{2}, \ldots, y_{r+2}\right) \\
& \leq-Q(k)\left(x_{1}-y_{1}\right)-\sum_{j=1}^{r} C_{j}(k)\left(x_{j+1}-y_{j+1}\right) \\
& \quad-q(k)\left(x_{r+2}-y_{r+2}\right)
\end{aligned}
$$

holds, where the functions $Q, q, C_{j}: \mathbb{Z}[a+1, T] \rightarrow$ $\mathbb{R}_{+}, j \in \mathbb{Z}[1, r]$, and inequality (8) is satisfied.

(3) The function $g \in \Lambda\left(\alpha_{0}, \beta_{0}\right)$.

(4) The functions $\tau_{j}(k): \mathbb{Z}[a+1, T] \rightarrow \mathbb{Z}[a+1-h, T]:$ $k-h \leq \tau_{j}(k) \leq k$ for $j \in \mathbb{Z}[1, r], k \in \mathbb{Z}[a+1, T]$.

Then there exist two sequences of functions $\left\{\alpha_{n}\right\}_{n=0}^{\infty}$ and $\left\{\beta_{n}\right\}_{n=0}^{\infty}$ such that

(a) The functions $\alpha_{n}, \beta_{n}: \mathbb{Z}[a-h+1, T] \rightarrow \mathbb{R},(n=1$, $2, \ldots$ ) form a couple of quasi lower and upper solutions of $B V P(1)-(2)$.

(b) For $k \in \mathbb{Z}[a-h+1, T]$ the inequalities

$$
\begin{aligned}
\alpha_{0}(k) & \leq \alpha_{1}(k) \leq \cdots \leq \alpha_{n}(k) \\
& \leq \cdots \leq \beta_{n}(k) \leq \cdots \leq \beta_{1}(k) \leq \beta_{0}(k)
\end{aligned}
$$

hold.

(c) Both sequences are convergent on $\mathbb{Z}[a-h+1, T]$ and their limits $\alpha(k)=\lim _{n \rightarrow \infty} \alpha_{n}(k)$ and $\beta(k)=$ $\lim _{n \rightarrow \infty} \beta_{n}(k)$ form a couple of quasi solutions of BVP (1)-(2) in $S\left(\alpha_{0}, \beta_{0}\right)$. In the case of uniqueness of the solution of BVP (1)-(2) in $S\left(\alpha_{0}, \beta_{0}\right)$ the equality $\alpha \equiv$ $\beta \equiv U$ holds, where $U$ is the unique solution of (1)-(2) in $S\left(\alpha_{0}, \beta_{0}\right)$.

Proof. Note if $u \in S\left(\alpha_{0}, \beta_{0}\right)$, then $\alpha_{0}\left(\tau_{j}(k)\right) \leq u\left(\tau_{j}(k)\right) \leq$ $\beta_{0}\left(\tau_{j}(k)\right), j \in \mathbb{Z}[1, r]$, and $\max _{s \in \mathbb{Z}[k-h, k]} \alpha_{0}(s) \leq$ $\max _{s \in \mathbb{Z}[k-h, k]} u(s) \leq \max _{s \in \mathbb{Z}[k-h, k]} \beta_{0}(s)$ for $k \in \mathbb{Z}[a+1, T]$. Therefore, according to condition 2 of Theorem 6 if the functions $u, v \in S\left(\alpha_{0}, \beta_{0}\right)$ satisfy $u(k) \leq v(k)$ for $k \in \mathbb{Z}[a-h+1, T]$, then for any $k \in \mathbb{Z}[a+1, T]$ the inequality

$$
\begin{aligned}
f(k, \Xi(u(\tau(k))))-f(k, \Xi(v(\tau(k)))) \\
\leq-Q(k)[u(k)-v(k)] \\
\quad-\sum_{j=1}^{r} C_{j}(k)\left[u\left(\tau_{j}(k)\right)-v\left(\tau_{j}(k)\right)\right] \\
\quad-q(k)\left[\max _{s \in \mathbb{Z}[k-h, k]} u(s)-\max _{s \in \mathbb{Z}[k-h, k]} v(s)\right]
\end{aligned}
$$

holds.

We will give an algorithm for construction of successive approximations to the unknown exact solution of BVP (1)(2).

Assume the functions $\alpha_{1}, \alpha_{2}, \ldots, \alpha_{n}: \mathbb{Z}[a-h+1, T] \rightarrow \mathbb{R}$ and $\beta_{1}, \beta_{2}, \ldots, \beta_{n}: \mathbb{Z}[a-h+1, T] \rightarrow \mathbb{R}$ are constructed such that

(H1) $\alpha_{j-1}(k) \leq \alpha_{j}(k), \beta_{j-1}(k) \geq \beta_{j}(k)$ for $j \in \mathbb{Z}[1, n], k \in$ $\mathbb{Z}[a-h+1, T]$

(H2) the functions $\alpha_{j}$ and $\beta_{j}, j \in \mathbb{Z}[1, n]$ form a couple of quasi lower and upper solutions of BVP (1)-(2);

(H3) $\alpha_{j}(k) \leq \beta_{j}(k), k \in \mathbb{Z}[a-h+1, T], j \in \mathbb{Z}[1, n] ;$

(H4) each function $\alpha_{j}, j \in \mathbb{Z}[1, n]$, is the unique solution of the initial value problem for the linear difference equation with maxima

$$
\begin{aligned}
& \Delta x(k-1) \\
& =P\left(k, \alpha_{j-1}(k)\right)-Q(k) x(k)-\sum_{l=1}^{r} C_{l}(k) x\left(\tau_{l}(k)\right) \\
& -q(k) \max _{s \in \mathbb{Z}[k-h, k]} x(s), \quad k \in \mathbb{Z}[a+1, T], \\
& x(a)=\alpha_{j-1}(a)-\frac{1}{\gamma} g\left(\alpha_{j-1}(a), \chi\left(\beta_{j}(T)\right)\right) \\
& x(k)=x(a), \quad k \in \mathbb{Z}[a-h+1, a-1],
\end{aligned}
$$

where $P: \mathbb{Z}[a+1, T] \times S\left(\alpha_{0}, \beta_{0}\right) \rightarrow \mathbb{R}$ is defined by

$$
\begin{aligned}
P(k, u(k)) & \\
= & f(k, \Xi(u(\tau(k))))+Q(k) u(k) \\
& +\sum_{j=1}^{r} C_{j}(k) u\left(\tau_{j}(k)\right)+q(k) \max _{s \in \mathbb{Z}[k-h, k]} u(s) ;
\end{aligned}
$$

(H5) each function $\beta_{j}, j \in \mathbb{Z}[1, n]$, is the unique solution of the initial value problem 
$\Delta x(k-1)$

$$
\begin{aligned}
& =P\left(k, \beta_{j-1}(k)\right)-\mathrm{Q}(k) x(k)-\sum_{l=1}^{r} C_{l}(k) x\left(\tau_{l}(k)\right) \\
& -q(k) \max _{s \in \mathbb{Z}[k-h, k]} x(s), \quad k \in \mathbb{Z}[a+1, T], \\
& x(a)=\beta_{j-1}(a)-\frac{1}{\gamma} g\left(\beta_{j-1}(a), \chi\left(\alpha_{j}(T)\right)\right), \\
& x(k)=x(a), \quad k \in \mathbb{Z}[a-h+1, a-1] .
\end{aligned}
$$

Now we will explain how to construct the next approximations $\alpha_{n+1}$ and $\beta_{n+1}$.

Consider the initial value problem (25) for $j=n$. According to Section 4 linear initial value problem (25) has a unique solution $\alpha_{n+1}: \mathbb{Z}[a-h+1, T] \rightarrow \mathbb{R}$.

Define a function $p_{1}: \mathbb{Z}[a-h+1, T] \rightarrow \mathbb{R}$ by $p_{1}(k)=$ $\alpha_{n}(k)-\alpha_{n+1}(k)$.

From claims (H2) and (H4) we get $p_{1}(k)=p_{1}(a)$ for $k \in$ $\mathbb{Z}[a-h+1, a-1]$ and $p_{1}(a)=(1 / \gamma) g\left(\alpha_{n}(a), \chi\left(\beta_{n}(T)\right)\right) \leq 0$.

Let $k \in \mathbb{Z}[a+1, T]$. Then from claims (H1), (H4), and inequality

$$
\begin{array}{r}
\max _{s \in \mathbb{Z}[k-h, k]} \alpha_{n}(s)-\max _{s \in \mathbb{Z}[k-h, k]} \alpha_{n+1}(s) \\
\geq \min _{s \in \mathbb{Z}[k-h, k]}\left(\alpha_{n}(s)-\alpha_{n+1}(s)\right)
\end{array}
$$

we get

$$
\begin{aligned}
\Delta p_{1}(k-1)= & -Q(k)\left[\alpha_{n}(k)-\alpha_{n+1}(k)\right] \\
& -\sum_{j=1}^{r} C_{j}(k)\left[\alpha_{n}\left(\tau_{j}(k)\right)-\alpha_{n+1}\left(\tau_{j}(k)\right)\right] \\
& -q(k)\left[\max _{s \in \mathbb{Z}[k-h, k]} \alpha_{n}(s)-\max _{s \in \mathbb{Z}[k-h, k]} \alpha_{n+1}(s)\right] \\
& +f\left(k, \Xi\left(\alpha_{n-1}(\tau(k))\right)\right)-f\left(k, \Xi\left(\alpha_{n}(\tau(k))\right)\right) \\
& +Q(k)\left[\alpha_{n-1}(k)-\alpha_{n}(k)\right] \\
& +\sum_{j=1}^{r} C_{j}(k)\left[\alpha_{n-1}\left(\tau_{j}(k)\right)-\alpha_{n}\left(\tau_{j}(k)\right)\right] \\
& +q(k)\left[\max _{s \in \mathbb{Z}[k-h, k]} \alpha_{n-1}(s)-\max _{s \in \mathbb{Z}[k-h, k]} \alpha_{n}(s)\right] \\
\leq & -Q(k) p_{1}(k)-\sum_{j=1}^{r} C_{j}(k) p_{1}\left(\tau_{j}(k)\right) \\
& -q(k) \min _{s \in \mathbb{Z}[k-h, k]} p_{1}(s) .
\end{aligned}
$$

According to Lemma $5 p_{1}(k) \leq 0$ for $k \in \mathbb{Z}[a-h+1, T]$. Hence, $\alpha_{n}(k) \leq \alpha_{n+1}(k)$ for $k \in \mathbb{Z}[a-h+1, T]$.

Consider the linear initial value problem (27) for $j=n$. According to Section 4 the linear initial value problems (27) has a unique solution $\beta_{n+1}: \mathbb{Z}[a-h+1, T] \rightarrow \mathbb{R}$.
Similarly as previously mentioned we prove $\beta_{n}(k) \geq$ $\beta_{n+1}(k)$ for $k \in \mathbb{Z}[a-h+1, T]$; that is, claim (H1) is satisfied for $j=n+1$.

Now we will prove $(\mathrm{H} 3)$ for $j=n+1$. Define a function $p_{2}: \mathbb{Z}[a-h+1, T] \rightarrow \mathbb{R}$ by $p_{2}(k)=\alpha_{n+1}(k)-\beta_{n+1}(k)$.

From claims (H2), (H3), and condition 3 of Theorem 6 we have $p_{2}(k)=p_{2}(a)$ for $k \in \mathbb{Z}[a-h+1, a-1]$. Also,

$$
\begin{aligned}
p_{2}(a)= & \alpha_{n}(a)-\beta_{n}(a) \\
& +\frac{1}{\gamma}\left(g\left(\beta_{n}(a), \chi\left(\alpha_{n}(T)\right)\right)-g\left(\alpha_{n}(a), \chi\left(\beta_{n}(T)\right)\right)\right) \\
\leq & \alpha_{n}(a)-\beta_{n}(a) \\
& +\frac{1}{\gamma}\left(g\left(\beta_{n}(a), \chi\left(\beta_{n}(T)\right)\right)-g\left(\alpha_{n}(a), \chi\left(\beta_{n}(T)\right)\right)\right)
\end{aligned}
$$$$
\leq 0 \text {. }
$$

Let $k \in \mathbb{Z}[a+1, T]$. From claims (H1), (H5), and inequality (24) we get

$$
\begin{aligned}
\Delta p_{2}( & -1) \\
= & f\left(k, \Xi\left(\alpha_{n}(\tau(k))\right)\right)-f\left(k, \Xi\left(\beta_{n}(\tau(k))\right)\right) \\
& +Q(k)\left[\alpha_{n}(k)-\beta_{n}(k)\right] \\
& +\sum_{j=1}^{r} C_{j}(k)\left(\alpha_{n}\left(\tau_{j}(k)\right)-\beta_{n}\left(\tau_{j}(k)\right)\right) \\
& +q(k)\left[\max _{s \in \mathbb{Z}[k-h, k]} \alpha_{n}(s)-\max _{s \in \mathbb{Z}[k-h, k]} \beta_{n}(s)\right] \\
& -Q(k) p_{3}(k)-\sum_{j=1}^{r} C_{j}(k) p_{3}\left(\tau_{j}(k)\right) \\
& -q(k)\left[\max _{s \in \mathbb{Z}[k-h, k]} \alpha_{n+1}(s)-\max _{s \in \mathbb{Z}[k-h, k]} \beta_{n+1}(s)\right] \\
\leq & -Q(k) p_{2}(k)-\sum_{j=1}^{r} C_{j}(k) p_{2}\left(\tau_{j}(k)\right) \\
& -q(k) \min _{s \in \mathbb{Z}[k-h, k]} p_{2}(s) .
\end{aligned}
$$

In view of Lemma 5 it follows $p_{2}(k) \leq 0$ for $k \in \mathbb{Z}[a-h+$ $1, T]$; that is, claim (H3) is satisfied for $j=n+1$.

Therefore, $\alpha_{n+1}, \beta_{n+1} \in S\left(\alpha_{0}, \beta_{0}\right)$.

We will prove $(\mathrm{H} 2)$ for $j=n+1$.

From condition 3 of Theorem 6 , claim (H2) for $j=n$ and the construction of functions $\alpha_{n+1}$ and $\beta_{n+1}$ we obtain

$$
\begin{aligned}
& \frac{1}{\gamma} g\left(\alpha_{n+1}(a), \chi\left(\beta_{n+1}(T)\right)\right) \\
& \quad \leq \frac{1}{\gamma} g\left(\alpha_{n+1}(a), \chi\left(\beta_{n}(T)\right)\right) \\
& \quad=\frac{1}{\gamma}\left(g\left(\alpha_{n+1}(a), \chi\left(\beta_{n}(T)\right)\right)-g\left(\alpha_{n}(a), \chi\left(\beta_{n}(T)\right)\right)\right)
\end{aligned}
$$




$$
\begin{aligned}
&+\frac{1}{\gamma} g\left(\alpha_{n}(a), \chi\left(\beta_{n}(T)\right)\right) \\
& \leq \alpha_{n+1}(a)-\alpha_{n}(a)+\frac{1}{\gamma} g\left(\alpha_{n}(a), \chi\left(\beta_{n}(T)\right)\right)=0, \\
& \frac{1}{\gamma} g\left(\beta_{n+1}(a), \chi\left(\alpha_{n+1}(T)\right)\right) \\
& \geq \frac{1}{\gamma} g\left(\beta_{n+1}(a), \chi\left(\alpha_{n}(T)\right)\right) \\
&=-\frac{1}{\gamma}\left(g\left(\beta_{n}(a), \chi\left(\alpha_{n}(T)\right)\right)-g\left(\beta_{n+1}(a), \chi\left(\alpha_{n}(T)\right)\right)\right) \\
&+\frac{1}{\gamma} g\left(\beta_{n}(a), \chi\left(\alpha_{n}(T)\right)\right) \\
& \geq \beta_{n+1}(a)-\beta_{n}(a)+\frac{1}{\gamma} g\left(\beta_{n}(a), \chi\left(\alpha_{n}(T)\right)\right)=0 .
\end{aligned}
$$

Let $k \in \mathbb{Z}[a+1, T]$. From claims (H1), (H4), and inequality (24) we get

$$
\begin{aligned}
\Delta \alpha_{n+1} & (k-1) \\
= & f\left(k, \Xi\left(\alpha_{n}(\tau(k))\right)\right)-Q(k)\left[\alpha_{n+1}(k)-\alpha_{n}(k)\right] \\
& -\sum_{j=1}^{r} C_{j}(k)\left[\alpha_{n+1}\left(\tau_{j}(k)\right)-\alpha_{n}\left(\tau_{j}(k)\right)\right] \\
& -q(k)\left[\max _{s \in \mathbb{Z}[k-h, k]} \alpha_{n+1}(s)-\max _{s \in \mathbb{Z}[k-h, k]} \alpha_{n}(s)\right] \\
= & f\left(k, \Xi\left(\alpha_{n+1}(\tau(k))\right)\right) \\
& +\left[f\left(k, \Xi\left(\alpha_{n}(\tau(k))\right)\right)-f\left(k, \Xi\left(\alpha_{n+1}(\tau(k))\right)\right)\right] \\
& -Q(k)\left[\alpha_{n+1}(k)-\alpha_{n}(k)\right] \\
& -\sum_{j=1}^{r} C_{j}(k)\left[\alpha_{n+1}\left(\tau_{j}(k)\right)-\alpha_{n}\left(\tau_{j}(k)\right)\right] \\
& -q(k)\left[\max _{s \in \mathbb{Z}[k-h, k]} \alpha_{n+1}(s)-\max _{s \in \mathbb{Z}[k-h, k]} \alpha_{n}(s)\right] \\
\leq & f\left(k, \Xi\left(\alpha_{n+1}(\tau(k))\right)\right) .
\end{aligned}
$$

Similarly, we prove

$$
\Delta \beta_{n+1}(k-1) \geq f\left(k, \Xi\left(\beta_{n+1}(\tau(k))\right)\right) .
$$

From inequalities (32), (33), and (34) it follows the functions $\alpha_{n+1}$ and $\beta_{n+1}$ form a couple of quasi lower and upper solution of BVP (1)-(2) on $\mathbb{Z}[a-h+1, T]$; that is, claim (H2) is satisfied for $j=n+1$.

Following the suggested previous scheme we can construct two sequences of functions $\left\{\alpha_{n}\right\}_{n=1}^{\infty}$ and $\left\{\beta_{n}\right\}_{n=1}^{\infty}$ which satisfy claims $(\mathrm{H} 1)-(\mathrm{H} 5)$.

For any fixed $k \in \mathbb{Z}[a-h+1, T]$ the sequences $\left\{\alpha_{n}\right\}_{n=0}^{\infty}$ and $\left\{\beta_{n}\right\}_{n=0}^{\infty}$ are monotone nondecreasing and monotone nonincreasing, respectively, and they are bounded by the functions $\alpha_{0}$ and $\beta_{0}$. Therefore, they are convergent; that is, there exist functions $\alpha, \beta: \mathbb{Z}[a-h+1, T] \rightarrow \mathbb{R}$ such that

$$
\begin{array}{r}
\lim _{n \rightarrow \infty} \alpha_{n}(k)=\alpha(k), \quad \lim _{n \rightarrow \infty} \beta_{n}(k)=\beta(k), \\
k \in \mathbb{Z}[a-h+1, T] .
\end{array}
$$

From claim (H3) it is clear that $\alpha, \beta \in S\left(\alpha_{0}, \beta_{0}\right)$. Also for any $k \in \mathbb{Z}[a+1, T]$ the equalities

$$
\begin{aligned}
& \lim _{n \rightarrow \infty}\left[\max _{m \in \mathbb{Z}[k-h, k]} \alpha_{n}(m)\right]=\max _{m \in \mathbb{Z}[k-h, k]}\left[\lim _{n \rightarrow \infty} \alpha_{n}(m)\right], \\
& \lim _{n \rightarrow \infty}\left[\max _{m \in \mathbb{Z}[k-h, k]} \beta_{n}(m)\right]=\max _{m \in \mathbb{Z}[k-h, k]}\left[\lim _{n \rightarrow \infty} \beta_{n}(m)\right]
\end{aligned}
$$

hold (for the proof see [19]).

We will prove that the limit $\alpha$ is a solution of BVP (1)-(2) on $\mathbb{Z}[a-h+1, T]$.

Taking a limit as $n \rightarrow \infty$ in the last equalities of (25) and (27) we get $\alpha(k)=\alpha(a)$ and $\beta(k)=\beta(a)$ for $k \in \mathbb{Z}[a-h+1, a-$ 1]. Also we obtain $\alpha(a)=\alpha(a)-(1 / \gamma) g(\alpha(a), \chi(\beta(T)))$ and $\beta(a)=\beta(a)-(1 / \gamma) g(\beta(a), \chi(\alpha(T)))$ or $g(\alpha(a), \chi(\beta(T)))=0$ and $g(\beta(a), \chi(\alpha(T)))=0$.

Taking a limit as $n \rightarrow \infty$ in the first equality of (25) and (27) we get the functions $\alpha$ and $\beta$ satisfy equality (1) for $k \epsilon$ $\mathbb{Z}[a+1, T]$.

Therefore, functions $\alpha$ and $\beta$ form a couple of quasi solutions of BVP (1)-(2) in $S\left(\alpha_{0}, \beta_{0}\right)$.

If nonlinear boundary value problem (1)-(2) has an unique solution $U$ in $S\left(\alpha_{0}, \beta_{0}\right)$, then $\alpha \equiv \beta \equiv U$.

\section{Applications}

Now we will give an example which exact solution will be obtained by a computer realization of the suggested previous algorithm. The example is a modification of the equation $u(k)=\left(C u^{2}(k-1)\right) /\left(u^{2}(k-1)+D\right)$ which is used in population biology to model a situation where the species survive if the initial size of population is greater than a certain threshold.

Example 7. Consider the nonlinear boundary value problem for the nonlinear difference equation with maxima

$$
\begin{array}{r}
\Delta u(k-1) \\
=\frac{0.1875(u(k))^{2}}{(u(k))^{2}+16}-0.05 u(\tau(k))-0.05 \max _{s \in \mathbb{Z}[k-3, k]} u(s), \\
\text { for } k \in \mathbb{Z}[3,10], \\
u(0)=u(1)=u(2), \quad u(2)-(u(2))^{2}+e^{u(10)}=0,
\end{array}
$$

where $\tau(k)=k-[\sqrt{k}], k \in \mathbb{Z}[3,10]$, and $[s]$ denotes the integer part of the real number $s$; that is,

$$
\tau(k)= \begin{cases}k-1 & k=3, \\ k-2, & k=4,5,6,7,8, \\ k-3, & k=9,10 .\end{cases}
$$


TABLE 1: Values of the successive lower/upper approximations $\alpha_{n}(k)$ and $\beta_{n}(k), n=0,1,2,3,4,5,6,7,8$.

\begin{tabular}{|c|c|c|c|c|c|c|c|}
\hline$k$ & 0 & 3 & 4 & 5 & $\cdots$ & 9 & 10 \\
\hline$\beta_{0}(k)$ & 0.300000 & 0.300000 & 0.300000 & 0.300000 & $\ldots$ & 0.300000 & 0.300000 \\
\hline$\beta_{1}(k)$ & 0.107373 & 0.102318 & 0.097378 & 0.092799 & $\ldots$ & 0.079779 & 0.077504 \\
\hline$\beta_{2}(k)$ & -0.161693 & -0.140751 & -0.121361 & -0.104382 & $\cdots$ & -0.054887 & -0.045805 \\
\hline$\beta_{3}(k)$ & -0.350522 & -0.313072 & -0.277815 & -0.246387 & $\cdots$ & -0.150852 & -0.132489 \\
\hline$\beta_{4}(k)$ & -0.450828 & -0.404758 & -0.361295 & -0.322444 & $\cdots$ & -0.203309 & -0.180134 \\
\hline$\beta_{5}(k)$ & -0.495330 & -0.445438 & -0.398367 & -0.356278 & $\cdots$ & -0.226976 & -0.201742 \\
\hline$\beta_{6}(k)$ & -0.513731 & -0.462242 & -0.413672 & -0.370244 & $\cdots$ & -0.236787 & -0.210719 \\
\hline$\beta_{7}(k)$ & -0.521346 & -0.469185 & -0.419987 & -0.376000 & $\cdots$ & -0.240816 & -0.214404 \\
\hline$\beta_{8}(k)$ & -0.524570 & -0.472122 & -0.422654 & -0.378427 & $\cdots$ & -0.242506 & -0.215947 \\
\hline$u(k)$ & -0.526 & -0.474 & -0.424 & -0.380 & $\cdots$ & -0.244 & -0.217 \\
\hline$\alpha_{8}(k)$ & -0.529472 & -0.476579 & -0.426694 & -0.382097 & $\cdots$ & -0.245037 & -0.218253 \\
\hline$\alpha_{7}(k)$ & -0.532445 & -0.479278 & -0.429136 & -0.384311 & $\cdots$ & -0.246548 & -0.219624 \\
\hline$\alpha_{6}(k)$ & -0.538843 & -0.485075 & -0.434371 & -0.389046 & $\cdots$ & -0.249751 & -0.222525 \\
\hline$\alpha_{5}(k)$ & -0.551920 & -0.496893 & -0.445013 & -0.398643 & $\cdots$ & -0.256153 & -0.228299 \\
\hline$\alpha_{4}(k)$ & -0.576447 & -0.518984 & -0.464830 & -0.416445 & $\cdots$ & -0.267835 & -0.238790 \\
\hline$\alpha_{3}(k)$ & -0.617154 & -0.555535 & -0.497512 & -0.445709 & $\cdots$ & -0.286810 & -0.255788 \\
\hline$\alpha_{2}(k)$ & -0.677874 & -0.610052 & -0.546283 & -0.489435 & $\cdots$ & -0.315694 & -0.281933 \\
\hline$\alpha_{1}(k)$ & -0.783286 & -0.704773 & -0.631632 & -0.567151 & $\cdots$ & -0.376223 & -0.340584 \\
\hline$\alpha_{0}(k)$ & -1.000000 & -1.000000 & -1.000000 & -1.000000 & $\cdots$ & -1.000000 & -1.000000 \\
\hline
\end{tabular}

Boundary value problem for the nonlinear delay difference equation with maxima (37)-(38) is from type (1)-(2), where $h=3, a=2, T=10, g(u, v)=u-u^{2}+e^{v}$, $f(k, x, y, z)=0.1875 x^{2} /\left(x^{2}+16\right)-0.05 y-0.05 z$.

The functions $\alpha_{0}(k)=-1$ and $\beta_{0}(k)=0.3, k \in \mathbb{Z}[0,10]$ form a couple of quasi lower and upper solutions of (37), (38), respectively, because $0 \geq\left(0.1875 * 0.3^{2}\right) /\left(0.3^{2}+16\right)-0.3 / 20-$ $0.3 / 20=-0.028951212,0 \leq 0.1875 /\left(1^{2}+16\right)+0.05+0.05=$ $0.111029,-1-(-1)^{2}+e^{0.3}=-0.65014119 \leq 0$, and $0.3-$ $(0.3)^{2}+e^{-1}=0.5778794 \geq 0$.

Consider the function $g(u, v)=u-u^{2}+e^{v}$ which is increasing in $v$. Let $u_{1}, u_{2}, v \in[-1,0.3]: u_{2} \leq u_{1}$. Then $g\left(u_{1}, v\right)-g\left(u_{2}, v\right)=\left(1-u_{1}-u_{2}\right)\left(u_{1}-u_{2}\right) \leq 3\left(u_{1}-u_{2}\right)$; that is, $\gamma=3$ and the boundary condition (38) is of antiperiodic type.

Let $x_{1}, x_{2}, y_{1}, y_{2}, z_{1}, z_{2} \in[-1,0.3], x_{1} \leq x_{2}, y_{1} \leq y_{2}, z_{1} \leq$ $z_{2}$. Then

$$
\begin{aligned}
f & \left(k, x_{1}, y_{1}, z_{1}\right)-f\left(k, x_{2}, y_{2}, z_{2}\right) \\
& =\frac{0.1875 x_{1}^{2}}{x_{1}^{2}+16}-\frac{0.1875 x_{2}^{2}}{x_{2}^{2}+16}-0.05\left(y_{1}-y_{2}\right)-0.05\left(z_{1}-z_{2}\right) \\
& =\frac{3\left(x_{1}-x_{2}\right)\left(x_{1}+x_{2}\right)}{\left(x_{1}^{2}+16\right)\left(x_{2}^{2}+16\right)}-0.05\left(y_{1}-y_{2}\right)-0.05\left(z_{1}-z_{2}\right) \\
& \leq \frac{-6\left(x_{1}-x_{2}\right)}{\left(x_{1}^{2}+16\right)\left(x_{2}^{2}+16\right)}-0.05\left(y_{1}-y_{2}\right)-0.05\left(z_{1}-z_{2}\right) \\
& \leq \frac{-3\left(x_{1}-x_{2}\right)}{128}-0.05\left(y_{1}-y_{2}\right)-0.05\left(z_{1}-z_{2}\right) ;
\end{aligned}
$$

that is, $q(k)=0.05, C(k)=0.05$, and $Q(k)=3 / 128$. Note that the inequality $(8)$ is satisfied because $\sum_{l=3}^{10}(3 / 128+0.05+$ $0.05)=0.9875<1$.

According to Theorem 6 the nonlinear boundary value problem (37)-(38) has a solution $u(k) \in[-1,0.3], k \in$ $\mathbb{Z}[0,10]$. We will obtain $u(k)$ as a limit of two sequences of successive approximations.

The approximation $\alpha_{n}, n=1,2, \ldots$, is a solution of the initial value problem for the linear delay difference equation with maxima (25) which is reduced to

$$
\begin{aligned}
\alpha_{n}(k)= & \alpha_{n}(k-1)-0.0234375 \alpha_{n}(k)-0.05 \max _{s \in \mathbb{Z}[k-3, k]} \alpha_{n}(s) \\
& -0.05 \alpha_{n}(\tau(k))+P\left(\alpha_{n-1}(k)\right), \quad k \in \mathbb{Z}[3,10],
\end{aligned}
$$

$\alpha_{n}(0)=\alpha_{n}(1)=\alpha_{n}(2)=\frac{2}{3} \alpha_{n-1}(2)+\frac{1}{3}\left(\alpha_{n-1}(2)\right)^{2}-\frac{1}{3} e^{\beta_{n-1}(10)}$,

and the approximation $\beta_{n}, n=1,2, \ldots$, is a solution of IVP (27) which is reduced to

$$
\begin{aligned}
\beta_{n}(k)= & \beta_{n}(k-1)-0.0234375 \beta_{n}(k)-0.05 \max _{s \in \mathbb{Z}[k-3, k]} \beta_{n}(s) \\
& -0.05 \beta_{n}(\tau(k))+P\left(\beta_{n-1}(k)\right), \quad k \in \mathbb{Z}[3,10], \\
\beta_{n}(0)= & \beta_{n}(1)=\beta_{n}(2) \\
= & \frac{2}{3} \beta_{n-1}(2)+\frac{1}{3}\left(\beta_{n-1}(2)\right)^{2}-\frac{1}{3} e^{\alpha_{n-1}(10)},
\end{aligned}
$$

where $P(u)=0.1875 u^{2} /\left(u^{2}+16\right)+0.0234375 u$.

The algorithm for solving linear problems (41) and (42) is given in Section 4. It is computer realized, and some of the results are given in Table 1 . 
From Table 1 it could be seen that the sequence $\left\{\alpha_{n}\right\}$ is increasing and the sequence $\left\{\beta_{n}\right\}$ is decreasing and $\alpha_{n}(k) \leq$ $\beta_{n}(k), k=\mathbb{Z}[0,10]$. The unknown exact solution $u(k) \in$ $\left[\alpha_{8}(k), \beta_{8}(k)\right], k \in \mathbb{Z}[0,10]$ and its approximate values are written in Table 1.

\section{Acknowledgments}

Research was partially supported by Project BG051PO001/ 3.3-05-001 Science and Business, financed by the Operative Program "Development of Human Resources," European Social Fund.

\section{References}

[1] R. P. Agarwal and S. Hristova, "Quasilinearization for initial value problems involving differential equations with "maxima'”, Mathematical and Computer Modelling, vol. 55, no. 9-10, pp. 2096-2105, 2012.

[2] M. J. Bohner, A. T. Georgieva, and S. G. Hristova, "Nonlinear differential equations with "maxima": parametric stability in terms of two measures," Applied Mathematics \& Information Sciences, vol. 7, no. 1, pp. 41-48, 2013.

[3] A. Golev, S. Hristova, and A. Rahnev, "An algorithm for approximate solving of differential equations with "maxima'”, Computers \& Mathematics with Applications, vol. 60, no. 10, pp. 2771-2778, 2010.

[4] S. Hristova and A. Golev, "Monotone iterative method for the initial value problem with initial time difference for differential equations with "maxima"' Abstract and Applied Analysis, vol. 2012, Article ID 493271, 17 pages, 2012.

[5] S. Hristova, A. Golev, and K. Stefanova, "Approximate method for boundary value problems of anti-periodic type for differential equations with "maxima'"' Boundary Value Problems, vol. 2013, 12 pages, 2013.

[6] D. Bainov and S. Hristova, Differential Equations with Maxima, Taylor \& Francis; CRC Press, 2011.

[7] A. Gelişken, C. Çinar, and A. S. Kurbanli, "On the asymptotic behavior and periodic nature of a difference equation with maximum," Computers \& Mathematics with Applications, vol. 59, no. 2, pp. 898-902, 2010.

[8] N. Touafek and Y. Halim, "On max type difference equations: expressions of solutions," International Journal of Nonlinear Sciences, vol. 11, no. 4, pp. 396-402, 2011.

[9] X. Yang, X. Liao, and C. Li, "On a difference equation wtih maximum," Applied Mathematics and Computation, vol. 181, no. 1, pp. 1-5, 2006.

[10] F. M. Atici, A. Cabada, and J. B. Ferreiro, "First order difference equations with maxima and nonlinear functional boundary value conditions," Journal of Difference Equations and Applications, vol. 12, no. 6, pp. 565-576, 2006.

[11] A. Cabada, V. Otero-Espinar, and R. L. Pouso, "Existence and approximation of solutions for first-order discontinuous difference equations with nonlinear global conditions in the presence of lower and upper solutions," Computers \& Mathematics with Applications, vol. 39, no. 1-2, pp. 21-33, 2000.

[12] Z. He and X. Zhang, "Monotone iterative technique for first order impulsive difference equations with periodic boundary conditions," Applied Mathematics and Computation, vol. 156, no. 3, pp. 605-620, 2004.
[13] C. C. Tisdell, "On first-order discrete boundary value problems," Journal of Difference Equations and Applications, vol. 12, no. 12, pp. 1213-1223, 2006.

[14] R. P. Agarwal, A. Cabada, and V. Otero-Espinar, "Existence and uniqueness results for $n$-th order nonlinear difference equations in presence of lower and upper solutions," Archives of Inequalities and Applications, vol. 1, no. 3-4, pp. 421-432, 2003.

[15] R. P. Agarwal, A. Cabada, V. Otero-Espinar, and S. Dontha, "Existence and uniqueness of solutions for anti-periodic difference equations," Archives of Inequalities and Applications, vol. 2, no. 4, pp. 397-411, 2004.

[16] T. Jankowski, "First-order functional difference equations with nonlinear boundary value problems," Computers \& Mathematics with Applications, vol. 59, no. 6, pp. 1937-1943, 2010.

[17] A. Cabada, "The method of lower and upper solutions for periodic and anti-periodic difference equations," Electronic Transactions on Numerical Analysis, vol. 27, pp. 13-25, 2007.

[18] P. Wang, S. Tian, and Y. Wu, "Monotone iterative method for first-order functional difference equations with nonlinear boundary value conditions," Applied Mathematics and Computation, vol. 203, no. 1, pp. 266-272, 2008.

[19] S. Hristova, A. Golev, and K. Stefanova, "Quasilinearization of the initial value problem for difference equations with "maxima'”' Journal of Applied Mathematics, Article ID 159031, 17 pages, 2012.

[20] R. Agarwal, S. Hristova, A. Golev, and K. Stefanova, "Monotone-iterative method for mixed boundary value problems for generalized difference equations with maxima," Journal of Applied Mathematics and Computing, vol. 2013, 2013.

[21] F. M. Atici, A. Cabada, and J. B. Ferreiro, "Existence and comparison results for first order periodic implicit difference equations with maxima," Journal of Difference Equations and Applications, vol. 8, no. 4, pp. 357-369, 2002. 


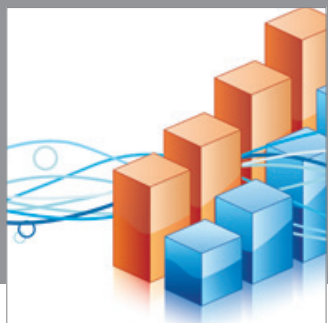

Advances in

Operations Research

mansans

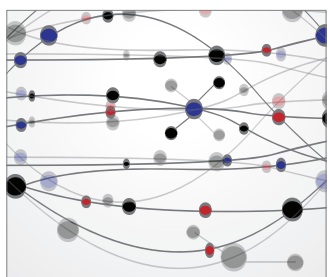

The Scientific World Journal
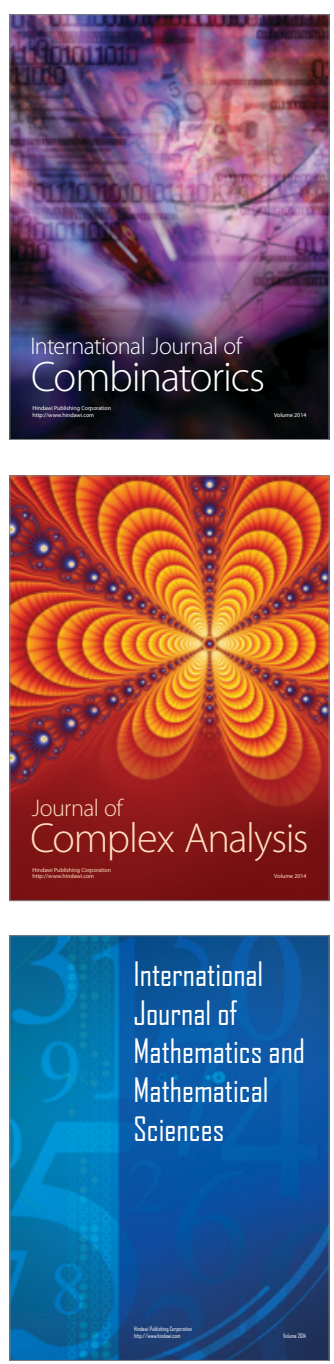
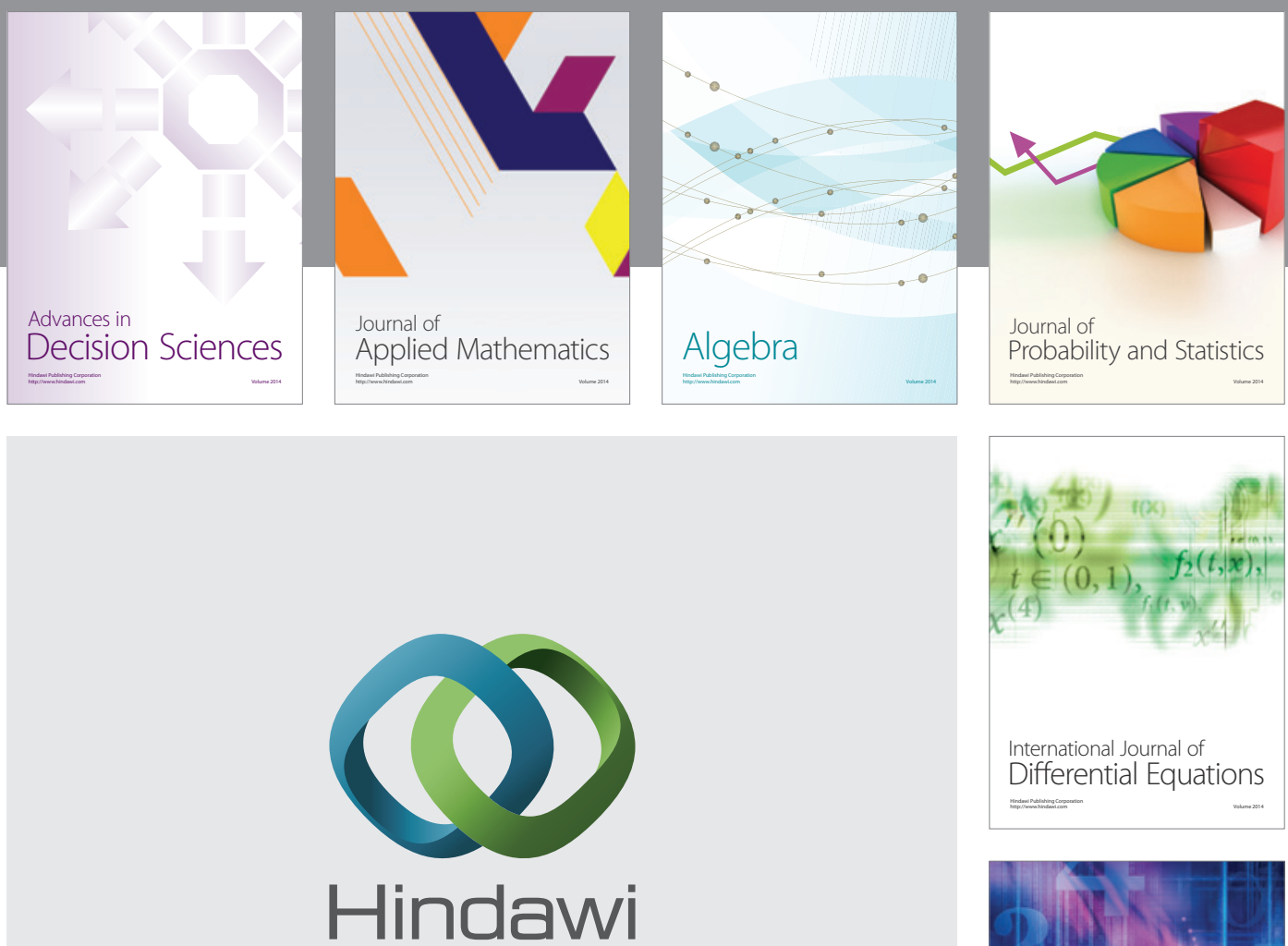

Submit your manuscripts at http://www.hindawi.com
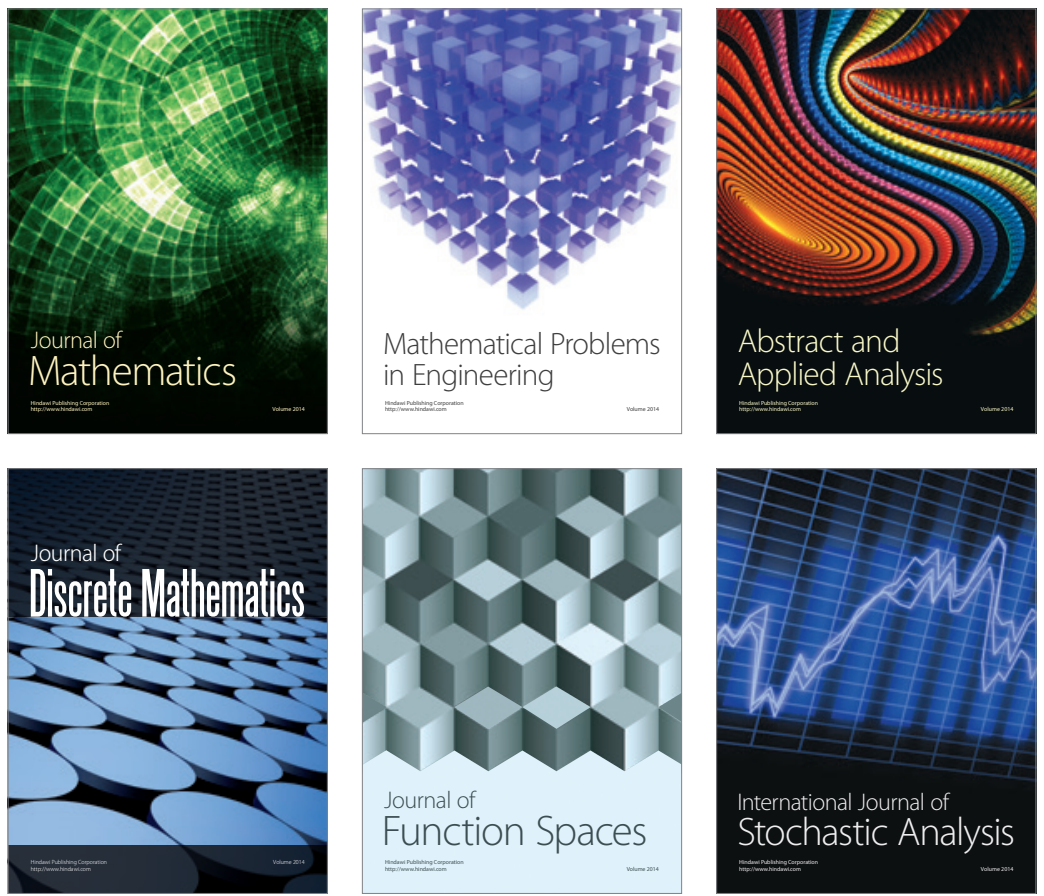

Journal of

Function Spaces

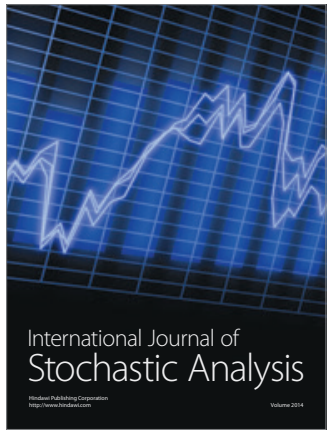

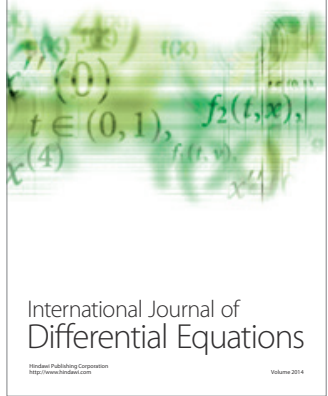
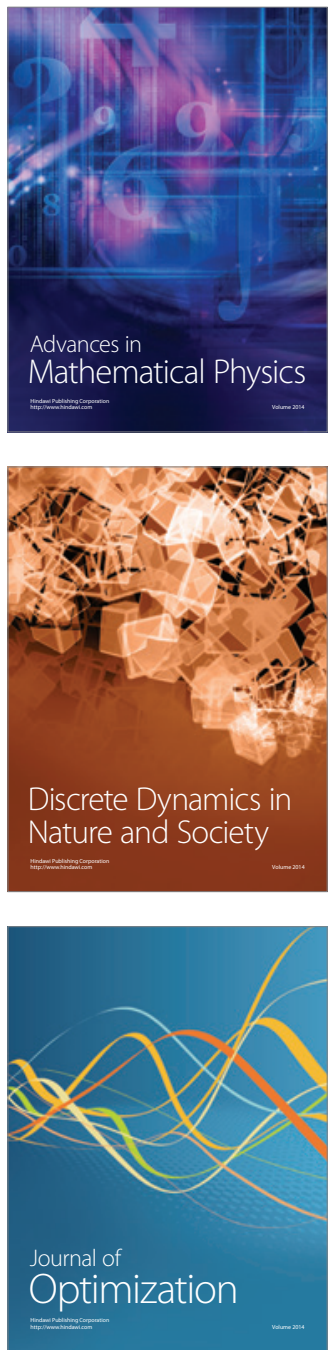